\title{
Multicast in Wireless Erasure Networks with Feedback
}

\author{
Chong Jiang, Brian Smith, Babak Hassibi, and Sriram Vishwanath
}

\begin{abstract}
This paper studies the lossy, wireless packet network of [1], in the case of a multicast requirement and the availability of feedback. In the unicast case, feedback is sufficient to allow a strategy which achieves the throughput-optimal cut-set capacity without requiring network coding [3]. We provide a counterexample to show that source coding and feedback, without network coding, is insufficient to achieve the cut-set capacity for the multicast wireless erasure network. In particular, we examine a network with one source, one relay, and two destinations. We show that even with the highly optimistic assumption of feedback which provides global packet state awareness, this network still fails to reach capacity. This bridges the gap between two previously known results; one, that network coding can achieve the capacity of the wireless erasure network, and two, that feedback allows a capacity achieving scheme which does not require network coding in the unicast wireless erasure network.
\end{abstract}

Index Terms-network information theory, erasure channel, feedback

\section{INTRODUCTION}

The wireless erasure network model, introduced in [1], incorporates the key features of a lossy, wireless packet network (packet losses and the broadcast nature of the wireless medium) while maintaining the analytic tractability of the erasure network [2]. The main result of [1] is that by using a strategy of linear network coding and allowing the final destination to have side-information concerning the location of packet erasures throughout the network, information can be reliably communicated from the source to several destination nodes at the information-theoretic min-cut max-flow rate. Further, although it is well known that feedback cannot increase the capacity of a discrete memoryless channel, various benefits of feedback may include an increase in the probability of error decoding exponent, or a decrease in coding complexity [4].

The simple erasure channel provides a straightforward example for which the availability of feedback eliminates the need for error-correction coding. The symmetric memoryless erasure channel consists of a simple conditional probability distribution: either a symbol from the input alphabet is seen at the receiver correctly with a probability $1-\epsilon$, or is "erased" ("dropped," or "lost") with a probability $\epsilon$, in which case the receiver sees an error symbol E. The capacity of such a channel is $1-\epsilon$. For this channel, the transmitter can send successive symbols to a receiver as long as they continue to

The first three authors would like to acknowledge funding from an ARO Young Investigator Award. The work of the third author was in parts supported by the National Science Foundation under grant CCF 0729203, by the David and Lucille Packard Foundation and by Caltech's Lee Center for Advanced Networking. be received correctly at the destination. The feedback allows the transmitter to know when a symbol is dropped or erased, and the transmitter can simply repeat the erased symbol until it is successfully received. It is straightforward to see that this is a capacity-achieving strategy.

It is shown in [3] that for a single-source, single-destination wireless erasure network, a randomized strategy exists by which feedback again eliminates the need for network coding. Specifically, with feedback which alerts each node in the network when a packet is received by the final destination, randomized routing alone will achieve the cut-set bound on capacity for the unicast wireless erasure network. It was this paper's goal to determine whether a similar statement could be made about the multicast case of the wireless erasure network with feedback.

Similar models of lossy packet networks were studied in [1], [5]. The first paper shows the achievability of the cutset bound in a multicast network with linear network coding; the second demonstrates a throughput-optimal backpressure strategy for multiple unicast networks in the multicommodity sense (that is, when network coding is disallowed) rather than in the information-theoretic sense.

This paper demonstrates with counter-examples that, even allowing for a rateless source coding scheme, routing without some form of network coding cannot in general achieve the min-cut rate of the multicast wireless erasure network.

\section{MOdEL AND PRELIMINARIES}

\section{A. Network Model}

Our basic network model is based on prior work by [1], [3]. The network is modeled as a directed acyclic graph $G=(\mathcal{V}, \mathcal{E})$, where each edge $\left(v_{i}, v_{j}\right) \in \mathcal{E}$ represents an memoryless erasure channel from $v_{i}$ to $v_{j}$ with erasure probability $\epsilon_{i j}$. The erasure events along each edge are i.i.d. over time, and independent from edge to edge. The network operates in a slotted-time manner: In each timeslot, every node may choose one of the packets which it has received in a prior timeslot to broadcast. The broadcast nature of the wireless medium is included by forcing the vertex $v_{i}$ to transmit the same symbol on all outbound edges. This is in contrast to traditional network models, such as that of [6], where a vertex can give each outgoing edge a unique symbol. We do not consider receiver interference, so for each timeslot, the vertex $v_{j}$ receives a vector of symbols, one from each $v_{i}$ where $\left(v_{i}, v_{j}\right) \in \mathcal{E}$. The network operates in multicast mode: that is, there will be a single source node and multiple destination nodes. The source node has a single message which all of the 
destinations are required to decode reliably. We will assume that each packet contains a header which uniquely identifies it.

We will consider the optimistic case in which every node in the network receives perfect delay-free feedback about the success and failure of each packet transmission attempt. That is, as soon as a attempt to send a packet is made, each node in the network will know whether it has been successfully received at each of the possible receivers. Thus, every node in the network knows the set of packets that any other node in the network has received. Note that this knowledge is only of the packet identifiers, and not of the packet contents. We will refer to this as knowledge of the global packet state.

In addition, when noted, we will allow a rateless source code to be applied to a set of $n$ information packets which we desire to reliably communicate to each destination node. The source node sends out a different coded packet during each timeslot. A key property of rateless codes is that for any $\delta_{1}>0$, when $n \rightarrow \infty$, a single destination is able to decode $n R$ packets with probability 1 as long as it receives at least $n R\left(1+\delta_{1}\right)$ unique coded packets [7], [8]. We say that a routing strategy can operate at a rate $R$ if it can transmit $n R$ packets in $n$ timeslots with probability 1 , as $n \rightarrow \infty$. A routing strategy on a network is said to be capacity-achieving if it can successfully operate at any rate $R=C-\varepsilon$, where $C$ is the information-theoretic capacity of the network.

Thus we will consider a multicast transmission to be successful as long as each of the destinations receives $n R\left(1+\delta_{1}\right)$ unique coded packets within $n$ timeslots, as the decoding at each node is independent. The overhead from the rateless coding header can be made arbitrarily small by taking the packet length arbitrarily large.

\section{B. Multicast Capacity of Wireless Erasure Networks}

The cut-set bound [4] is a general upper-bound on the rate of reliable communication in information networks. Intuitively, a cut-set upper-bound is obtained by partitioning the nodes of a network into two sets, and allowing all nodes in each partition to fully cooperate and use each others' inputs as side information. The rate at which information can be communicated from one side of the partition to the other is then clearly an upperbound on the sum-rate that information can be transmitted from sources on the first side to destinations on the other, in the actual network.

Following the convention in [9], define an $x-y$ cut for $x, y \in \mathcal{V}$ to be a partition of $\mathcal{V}$ into an $x$-set $\mathcal{V}_{x}$ and a $y$-set $\mathcal{V}_{y}=\mathcal{V}_{x}^{c}$, such that $x \in \mathcal{V}_{x}$ and $y \in \mathcal{V}_{y}$. If $s$ is the source node, and $d_{l}$ is one of the destination nodes, then the cut-capacity corresponding to any $s-d_{l}$ cut represented by the $s-$ set $\mathcal{V}_{s}$ and the destination set $\mathcal{V}_{d_{l}}=\mathcal{V}_{s}^{c}$ containing $d_{l}$ is then denoted by $C\left(\mathcal{V}_{s^{-} d_{l}}\right)$, where

$$
C\left(\mathcal{V}_{s-d_{l}}\right)=\sum_{i \in \mathcal{V}_{s}}\left(1-\prod_{j \in \mathcal{V}_{d_{l}}} \epsilon_{i j}\right),
$$

and where we take $\epsilon_{i j}$ to be 1 when $(i, j) \notin \mathcal{E}$.
The min-cut max-flow capacity of a single-source multicast network, from $s \in \mathcal{V}$ to $\mathcal{D}=\left\{d_{1}, d_{2}, \ldots, d_{|\mathcal{D}|}\right\} \subset \mathcal{V}$, is then given by

$$
C=\min _{d_{l} \in \mathcal{D}} \min _{\mathcal{V}_{s^{-} d_{l}}: s^{-} d_{l}} C\left(\mathcal{V}_{s^{-} d_{l}}\right) .
$$

In corollary 1 of [1], it is shown that this capacity is in fact achievable through linear network coding.

\section{RESUltS}

We begin by examining a simple network, where forwarding of packets without any coding is unable to achieve the min-cut rate when feedback is available. Consider the network show in Figure 1, with a single source $s$ and a broadcasting link to two destinations $d_{1}$ and $d_{2}$.

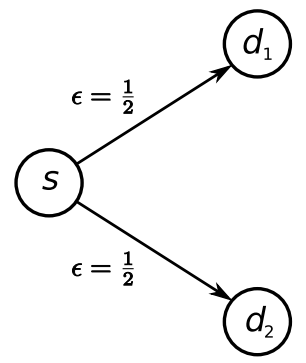

Figure 1. The counter-example network considered in Propositions 1 and 2. The min-cut upper-bound on capacity is $\frac{1}{2}$, which in Proposition 1 , we show cannot be achieved with routing and feedback alone. In Proposition 2 , we show that the combination of routing, feedback, and source coding is sufficient to achieve this capacity.

Proposition 1: There does not exist a routing-only strategy for the network shown in Figure 1 that is capacity-achieving.

Proof: Without the ability to code packets at $s$, all possible transmission strategies are equivalent to the strategy of a point-to-point erasure channel: transmit each packet until it is received by all intended destinations. As the channels are memoryless, all permutations on the order in which packets are sent are equivalent. The rate of this strategy can then be computed as $\frac{1}{T}$, where $T$ denotes the expected number of timeslots required to transmit a single packet.

Consider the transmission of a single packet, initially present at $s$. With a probability of $\frac{1}{4}$, both destinations receive the packet. With a probability of $\frac{1}{2}$, only one destination receives the packet. With a probability of $\frac{1}{4}$, neither destination receives the packet. Note that if only one destination receives the packet, the number of timeslots required for the other destination to receive the packet follows an geometric distribution with parameter $\frac{1}{2}$, and therefore has expected value 2 . Thus,

$$
T=\frac{1}{4}(1)+\frac{1}{2}(1+2)+\frac{1}{4}(1+T) .
$$

Solving the equation yields $T=\frac{8}{3}$, and therefore the achievable rate of this network is

$$
\frac{1}{T}=\frac{3}{8}<\frac{1}{2}=C \text {. }
$$

Thus routing alone is insufficient for this network to achieve capacity. 
Theorem 1: For a general multicast network under our network model, there does not exist a capacity-achieving routing scheme, in the absence of source coding.

Proof: This is a direct result of the counter-example in Proposition 1.

If we allow a rateless source code to be implemented, it is straightforward to see that the multicast capacity of $\frac{1}{2}$ packets/channel use can be achieved in the network of Figure 1.

Proposition 2: There exists a scheme with rateless source coding and routing-only for intermediate nodes for the network shown in Figure 1 that is capacity-achieving.

Proof: By implementing a rateless source code, the source node can transmit $n$ unique packets, one in each timeslot. For a $\delta_{2}$-typical sequence of erasures, at least $\frac{n}{2}\left(1-\delta_{2}\right)$ packets will be received with probability arbitrarily close to 1 , for sufficiently large $n$. To achieve a rate $R$, the destinations must each receive $n R\left(1+\delta_{1}\right)$ source-coded packets. Thus

$$
\begin{aligned}
n R\left(1+\delta_{1}\right) & \leq \frac{n}{2}\left(1-\delta_{2}\right) \\
R & \leq \frac{1}{2} \cdot \frac{1-\delta_{2}}{1+\delta_{1}} .
\end{aligned}
$$

The supremum over all achievable rates $R$, as $\delta_{1}, \delta_{2} \rightarrow 0$, is $\frac{1}{2}$, the multicast capacity of this network.

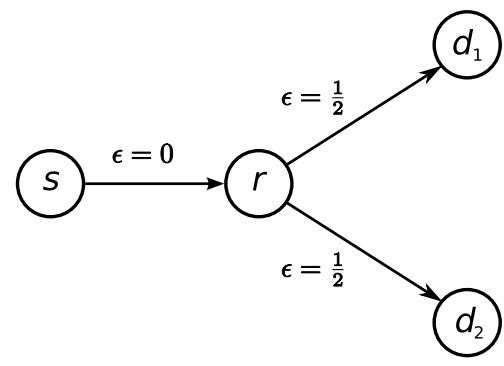

Figure 2. An example network which is equivalent to the network shown in Figure 1. The capacity-achieving network coding result of [1] applies here, and this network coding scheme for node $r$ here can be used as the source coding scheme for node $s$ in Figure 1 .

Alternatively, we can interpret source coding in the example of Figure 1 as a special case of the general wireless erasure network. Consider the network of Figure 2. This network is constructed by taking the network of Figure 1, and adding an additional lossless link from a new source node $s$ to the node $r$ (which previously acted as the source node). From [1], we know that a network coding scheme exists which obtains capacity for the network in Figure 2. Since the source node of Figure 1 has knowledge of everything that the relay node of the network in Figure 2 can ever have access to, it can implement the network coding scheme for obtaining capacity in Figure 2 as its own source coding scheme. Therefore, the upper-bound is achieved in Figure 1.

This result, namely, that source coding alone allows a capacity-achieving scheme for the network of Figure 1, inspires the conjecture that perhaps the combination of rate- less source coding and feedback is sufficient for a capacityachieving scheme for multicast wireless erasure networks in general. That is, it might be conjectured that network coding is not required to achieve the multicast capacity of wireless erasure networks, in the presence of feedback and source coding. We demonstrate with the counter-example network of Figure 3 that this conjecture is untrue. While a network coding scheme such as that in [1] can achieve the unicast and multicast capacity without feedback, and feedback obviates the need for coding in a unicast network [3], feedback and source coding are not sufficient to eliminate the need for network coding in a capacity-achieving scheme for the multicast case.

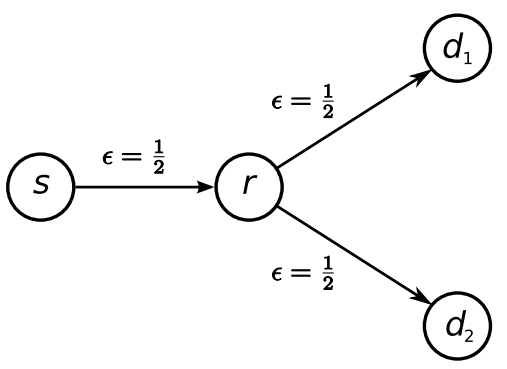

Figure 3. The counter-example network considered in Proposition 3. The min-cut upper-bound on capacity is $\frac{1}{2}$, which in Proposition 3 , we show cannot be achieved even with the combination of routing, feedback, and rateless source coding.

Proposition 3: There does not exist a routing-only scheme for the network shown in Figure 3 that is capacity-achieving, even when source coding is allowed.

Proof: Consider the network shown in Figure 3. To establish the multicast capacity for this network, we can apply (1) to obtain $C=\frac{1}{2}$. This max-flow min-cut upper-bound is achievable through linear network coding [1] when side information on erasure locations is available to the destinations. We examine the set of possible strategies for routing packets, and discover that no strategy allows a sufficient number of unique packets to arrive at both destination $d_{1}$ and $d_{2}$ simultaneously to decode at a rate of $\frac{1}{2}$. We incorporate a throughput-optimal source code, as well as knowledge of the global packet state.

For the network of Figure 3, there are 4 possible states for the packets present at the relay node $r$. Packets which have arrived at neither $d_{1}$ or $d_{2}$, as determined by the feedback, will be labeled as "00" states. Packets which have arrived at $d_{1}$ only are labeled "10", those whch have arrived at $d_{2}$ only are labeled "01", and those which have arrived at both destinations are labeled "11". Let $p_{i}$ denote the empirical fraction of the timeslots in which the sent packet was in state $i$ immediately prior to the transmission. A timeslot in which no packet is selected to be sent is defined to send a "11" packet, as the result in both cases is the same, with no new information reaching either destination.

Suppose the source node $s$ is sending packets using a rateless source code. In this case, achieving a rate of $R$ amounts to each destination receiving $n R\left(1+\delta_{1}\right)$ coded packets in $n$ timeslots. Suppose the erasures over each link are $\delta_{2}$-typical sequences. The number of innovative packets received at $d_{1}$ in 
$n$ timeslots is the sum of successfully received " 00 " and " $01 "$ packets, as only those are innovative to $d_{1}$. The total number of innovative packets received at $d_{1}$ will then be lower-bounded by

$$
\frac{n}{2}\left(p_{00}+p_{01}\right) \cdot\left(1-\delta_{2}\right) \text {. }
$$

If we assume the rate $R$ is achievable, then we expect this to exceed $n R\left(1+\delta_{1}\right)$. Thus

$$
p_{00}+p_{01} \geq \frac{2 R\left(1+\delta_{1}\right)}{\left(1-\delta_{2}\right)} .
$$

By the same argument for $D_{2}$,

$$
p_{00}+p_{10} \geq \frac{2 R\left(1+\delta_{1}\right)}{\left(1-\delta_{2}\right)} .
$$

As the four packet states together form the set of all possible actions at each timeslot,

$$
p_{00}+p_{01}+p_{10}+p_{11}=1 \text {. }
$$

Taking (2) + (3) - (4) yields

$$
p_{00} \geq \frac{4 R\left(1+\delta_{1}\right)}{\left(1-\delta_{2}\right)}-1 .
$$

Now consider the upper-bound on $p_{00}$. Let us define these "00" packets which have arrived from $s$ to $r$ to be original "00" packets. As the link from $s$ to $r$ also observes an $\delta_{2}$ typical sequence of erasures, the number of original packets is upper-bounded by $\frac{n\left(1+\delta_{2}\right)}{2}$. Note each original "00" packet can be sent a random number of times, following an geometric distribution with parameter $1-\left(\frac{1}{2}\right)^{2}$. As the packet selection scheme is memoryless, the expected number of timeslots in which a "00" packet is sent is equal to the number of original " 00 " packets multiplied by the expected number of transmissions a single original " 00 " packet undergoes. Thus,

$$
\begin{aligned}
p_{00} \cdot n & \leq \frac{n}{2}\left(1+\delta_{2}\right) \cdot \frac{1}{1-\left(\frac{1}{2}\right)^{2}} \\
p_{00} & \leq\left(1+\delta_{2}\right) \cdot \frac{2}{3} \\
\frac{4 R\left(1+\delta_{1}\right)}{\left(1-\delta_{2}\right)}-1 & \leq\left(1+\delta_{2}\right) \cdot \frac{2}{3} \\
R & \leq\left(\left(1+\delta_{2}\right) \cdot \frac{2}{3}+1\right) \cdot \frac{\left(1-\delta_{2}\right)}{4\left(1+\delta_{1}\right)} \\
& <\frac{5}{12} \cdot \frac{1-\left(\delta_{2}\right)^{2}}{1+\delta_{1}} .
\end{aligned}
$$

The supremum over all achievable rates $R$, as $\delta_{1}, \delta_{2} \rightarrow 0$, is $\frac{5}{12}$.

This upper-bound on the achievable rate for this network using any routing-only scheme, is $\frac{5}{12}$, which is strictly less than the multicast capacity $C=\frac{1}{2}$.

Theorem 2: For a general multicast network under our network model, there does not exist a capacity-achieving routing scheme, even when source coding is introduced.
Proof: This is a direct result of the counter-example in Proposition 3.

\section{DISCUSSION}

We have chosen two counter-examples to demonstrate that, while there exist strategies which eliminate the requirement for any sort of coding in a unicast wireless erasure network [3], network coding is required to achieve the multicast capacity.

Intuitively, the example of Figure 3 is a direct consequence of the concept behind Figure 1: without some sort of coding (rateless source coding, for example) at the source node in Figure 1, simple retransmission of the packets we intend to send is essentially the only strategy, and we have demonstrated that the retransmission strategy cannot achieve capacity. The network of Figure 3, from the relay node $r$ on and disallowing network coding, is equivalent to the network of Figure 1, except new unique packets arrive at the relay $r$ at a rate of $\frac{1}{2}$ instead of 1 . We have seen that this reduced arrival rate yields an insufficient number of unique packets at the destinations, thereby preventing successful decoding.

We have demonstrated in this paper that rates arbitrarily close to $\frac{5}{12}$ are achievable in the presence of source coding and feedback, but without network coding, for the network in Figure 3. A question for future research to ask would be if there is, in general, a tight bound on the achievable throughput of wireless erasure networks which allow source coding and feedback. Alternatively, how much does source coding and feedback improve the throughput rate of a wireless erasure network over a strategy of routing alone?

The wireless erasure network model of this paper considers the key wireless characteristics of a broadcast constraint, and the dropping of packets in a lossy environment. In this paper, we have determined that network coding, in general, is required in order to achieve the min-cut capacity of such multicast networks.

\section{REFERENCES}

[1] A. Dana, R. Gowaikar, R. Palanki, B. Hassibi, and M. Effros, "Capacity of wireless erasure networks," IEEE Trans. Inform. Theory, vol. 52, no. 3, pp. 789-804, Mar. 2006.

[2] D. Julian, "Erasure networks," in Proc. IEEE ISIT 2002, Lausanne, Switzerland, Jul. 2002. pp. 789-804, Mar. 2006.

[3] B. Smith and B. Hassibi, "Wireless erasure networks with feedback," in Proc. IEEE ISIT 2008, Toronto, Canada, Jul. 2008.

[4] T. Cover and J. Thomas, Elements of Information Theory. New York: Wiley, 1991.

[5] M. J. Neely and R. Urgaonkar, "Optimal Backpressure Routing in Wireless Networks with Multi-Receiver Diversity," in Proc. Conference on Information Sciences and Systems, Mar. 2006.

[6] R. Ahlswede, N. Cai, S.-Y. R. Li, and R. W. Yeung, "Network information flow," IEEE Trans. Inform. Theory, vol. 46, no. 4, pp. 1204-1216, Jul. 2000.

[7] A. Shokrollahi, "Raptor Codes," IEEE/ACM Trans. Netw., vol. 14, pp. 2551-2567, 2006

[8] M. Luby, "LT Codes," in Proc. 43rd Annual IEEE Symp. on Found. Comp. Sci. pp. 271-280, 2002.

[9] D. S. Lun, M. Médard, and M. Effros, "On coding for reliable communication over packet networks," in Proc. 42nd Annual Allerton Conf. on Commun. Control, and Computing, Sept. 2004. 